\title{
Polysaccharides from Bletilla striata: Extraction, Optimization and Their Antioxidant Activities in vitro
}

\author{
Xiaojing Shen ${ }^{a, b, c}$ and Weiwei Jiang ${ }^{*, a, d, e}$ \\ ${ }^{a}$ College of Science, Yunnan Agricultural University, Kunming, Yunnan 650201, China \\ ${ }^{b}$ Party Committee of Organ, Yunnan Agricultural University, Kunming, Yunnan 650201, China \\ ${ }^{c}$ College of Food Science and Technology, Yunnan Agricultural University, Kunming, Yunnan 650201, China \\ ${ }^{d}$ National \& Local Joint Engineering Research Center on Germplasm Innovation \& Utilization of Chinese \\ Medicinal Materials in Southwestern China, Kunming, Yunnan 650201, China \\ ${ }^{e}$ Key Laboratory of Medicinal Plant Biology, Yunnan Agricultural University, Kunming, Yunnan 650201, China
}

Email: 2015031@ynau.edu.cn (W.J.)

\begin{abstract}
In this paper, the extraction conditions of water-soluble polysaccharide from Bletilla striata were optimized by response surface methodology. With the dried Bletilla striata as raw materials, phenol-sulfuric acid method as the method of polysaccharide extraction rate, polysaccharide extraction rate as an indicator, the optimum process conditions for polysaccharide extraction from Bletilla striata were obtained including liquid-solid ratio of $15 \mathrm{mg} / \mathrm{mL}$, extraction temperature $90{ }^{\circ} \mathrm{C}$, extraction time of $90 \mathrm{~h}$, sample immersion time $90 \mathrm{~min}$, and extraction 2 times, achieving the corresponding polysaccharide yield of $48.38 \%$. The antioxidant assays in vitro revealed that the polysaccharides from Bletilla striata exhibited a slight scavenging activity for 1,1-diphenyl-2picrylhydrazyl radicals and 2,2'-azino-bis(3-ethylbenzothiazoline-6-sulfonic acid) radical cations.
\end{abstract}

Keywords Bletilla striata, polysaccharides, extraction, antioxidant activities

\section{Introduction}

Bletilla striata is a health food, which is the dried tuber of Bletilla Striata (Thunb.) Reichb.f and has a long medicinal history. ${ }^{[1]}$ It is mainly distributed in Guizhou, Sichuan, Hunan, Jiangxi and Guangxi provinces in China. Bletilla striata is also a traditional Chinese medicine, which is widely used by Chinese. It has several effects of astringency and hemostasis, clearing heat and promoting diuresis, detumescence and promoting granulation. Its pharmacological activities mainly include anti-bacterial, hemostasis, anti-tumor, anti-ulcer, anti-fibrosis, anti-oxidation, promoting wound healing, etc. Thus, Bletilla striata is widely used in clinic to treat hemoptysis, hematemesis, trauma bleeding, sores and swelling poison, skin chapping, anorectal diseases, gynecological fibroids, tumor embolism and prostate surgery, and so on. ${ }^{[2-8]}$ Bletilla striata contains several phytochemical compounds such as phenolic compounds, dihydrophenanthrene, phenanthrene, bibenzyl and polysaccharides. ${ }^{[9]}$ Polysaccharides are a class of macromolecular compounds that contain more than ten monosaccharides, and they are polymerized by glycosidic bonds. Polysaccharides commonly exist in animals, plants, microorganisms and algae. Some studies have confirmed polysaccharides possess various bioactivities including antioxidant, antitumor and immunoregulatory activities. ${ }^{[10-13]}$

This present work was aimed to optimize the extraction conditions for maximum yield of Bletilla striata polysaccharide (BSP) using response surface methodology and evaluate their in vitro antioxidant activity.

\section{Materials and Methods}

\section{Meterials and chemicals}

Bletilla striata was purchased from Liangbao Medicinal Materials Company in Puer city (Yunnan, China) in 2017. The dry Bletilla striata was crushed with a pulverizer and the $60-80$ mesh portion was taken as the experimental material. Ascorbic acid (Vc), 1,1-diphenyl-2-picrylhydrazyl radicals (DPPH) and 2,2'-azino-bis(3-ethylbenzothiazoline-6-sulfonic acid) (ABTS) were obtained from Solarbio Co., Ltd. (Beijing, China). All the reagents were of analytical grade.

\section{Extraction procedure}

$1.5 \mathrm{~g}$ (dry) of Bletilla striata power was placed in a $250 \mathrm{~mL}$ Erlenmeyer flask. It was extracted with hot water under the designated liquid-solid ratio, extraction temperature, extraction time, sample immersion time, extraction times and ethanol concentration. After extraction and filtration, the supernatant was concentrated to one quarter of the original volume and then precipitated by the addition of 4 volumes of absolute ethanol overnight at $4{ }^{\circ} \mathrm{C}$. The precipitates were washed by anhydrous alcohol three times. Afterwards, the crude polysaccharides samples were dried using drying cabinet at $35^{\circ} \mathrm{C}$.

\section{Single factor experiment design}

The effects of liquid-solid ratio, extraction times, ethanol concentration, sample immersion time, extraction time, and extraction temperature on the yield of BSP were performed using single-factor experimental design, where one factor was changed while others were kept constant. Each experiment was carried out three times in parallel.

\section{Effects of extraction method on extraction rate of BSP}

Under the conditions of extraction temperature $50{ }^{\circ} \mathrm{C}$, sample immersion time of $60 \mathrm{~min}$, ethanol concentration of $70 \%$, extraction of 1 time and the ratio of water to raw material of 15 $\mathrm{mL} / \mathrm{g}$, ultrasonic and heated reflux were used for extraction. The extraction time was 20,30 and 40 min, respectively. 


\section{Effects of liquid-solid ratio on extraction rate of BSP}

Under the conditions of extraction temperature $50{ }^{\circ} \mathrm{C}$, sample immersion time of $60 \mathrm{~min}$, extraction time of $30 \mathrm{~min}$, ethanol concentration of $70 \%$ and extraction of 1 time, the ratios of water to raw materials were $15,20,25,30 \mathrm{~mL} / \mathrm{g}$, respectively.

\section{Effects of extraction times on extraction rate of BSP}

Under the conditions of extraction temperature $50{ }^{\circ} \mathrm{C}$, sample immersion time of $60 \mathrm{~min}$, extraction time of $30 \mathrm{~min}$, ethanol concentration of $70 \%$ and the ratio of water to raw material of $15 \mathrm{~mL} / \mathrm{g}$, extraction times were $1,2,3$ and 4 , respectively.

\section{Effects of ethanol concentration on extraction rate of BSP}

Under the conditions of extraction temperature $50{ }^{\circ} \mathrm{C}$, sample immersion time of $60 \mathrm{~min}$, extraction time of $30 \mathrm{~min}$, extraction 1 time and the ratio of water to raw material of 15 $\mathrm{mL} / \mathrm{g}$, ethanol concentrations were $60 \%, 70 \%, 80 \%, 90 \%$, respectively.

\section{Effects of sample immersion time on extraction rate of BSP}

Under the conditions of extraction temperature $50{ }^{\circ} \mathrm{C}$, extraction time of $30 \mathrm{~min}$, ethanol concentration of $70 \%$, extraction 1 time and the ratio of water to raw material of 15 $\mathrm{mL} / \mathrm{g}$, sample immersion time was $30,60,90$ and $120 \mathrm{~min}$, respectively.

\section{Effects of extraction time on extraction rate of BSP}

Under the conditions of extraction temperature $50{ }^{\circ} \mathrm{C}$, sample immersion time of $60 \mathrm{~min}$, ethanol concentration of $70 \%$, extraction 1 time and the ratio of water to raw material of 15 $\mathrm{mL} / \mathrm{g}$, extraction time was $30,60,90$ and $120 \mathrm{~min}$, respectively.

\section{Effects of extraction temperature on extraction rate of BSP}

Under the conditions of sample immersion time of $60 \mathrm{~min}$, extraction time of $30 \mathrm{~min}$, ethanol concentration of $70 \%$, the ratio of water to raw material of $15 \mathrm{ml} / \mathrm{g}$ and extraction 1 time, extraction temperature was $50,60,70,80,90,95{ }^{\circ} \mathrm{C}$, respectively.

\section{Design of Box-Behnken}

Through the single-factor experimental, response surface methodology (RSM) was used to further optimize the experimental parameters. From Table 1, Box-Behnken design (BBD) of BSP was performed with three independent variables extraction temperature $(A)$, extraction time $(B)$, sample immersion time $(C)$ at three levels. The entire design consisted of 17 experimental runs, each of which was carried out in triplicate. The three variables at the three levels were coded as $-1,0$ and 1 . The total sugar content was measured by the phenol-sulfuric method using glucose as the standard.

\section{Antioxidant Activity in vitro}

\section{DPPH free radical scavenging activity}

The DPPH free radical scavenging activity was detected according to the previous literature with some modifications. Ascorbic acid (Vc) was used as the positive control. Briefly, DPPH ethanol solution $(4 \mathrm{~mL}, 0.0035 \mathrm{mg} / \mathrm{mL})$ was added into $2.00 \mathrm{~mL}$ BSP solution at varying concentrations $(0.2,0.4,0.6$, $0.8,1.0,2.0,3.0,4.0$ and $5.0 \mathrm{mg} / \mathrm{mL}$ ). The mixture was shaken thoroughly and incubated at room temperature in the dark environment for $30 \mathrm{~min}$. The absorbance of the sample was determined at $517 \mathrm{~nm}$. The solution of $2.00 \mathrm{~mL}$ deionized water and $4.00 \mathrm{~mL}$ absolute ethanol was blank control. ${ }^{[14-16]}$ The free radical scavenging activity was concluded by the following equation:

Scavenging rate $(\%)=\left[1-\left(A_{1}-A_{2}\right) / A_{0}\right] \times 100 \%$

where $A_{0}$ is the absorbance of the control, $A_{1}$ is the absorbance of the BSP sample, and $A_{2}$ is the absorbance of the BSP sample under similar condition to $A_{1}$ excepting ethanol instead of the DPPH.

\section{ABTS radical cation scavenging activity}

The ABTS radical scavenging of BSP was carried out referring the method of Wang. ${ }^{[14-16]} V_{c}$ was used as the positive control. The ABTS radical cation $\left(\mathrm{ABTS}^{*+}\right)$ was produced via the reaction between ABTS $(5 \mathrm{~mL}, 7 \mathrm{mM})$ and $\left(\mathrm{NH}_{4}\right)_{2} \mathrm{~S}_{2} \mathrm{O}_{8}$ aqueous solution ( $1 \mathrm{~mL}, 15 \mathrm{mM})$ following $12 \mathrm{~h}$ in the dark. The ABTS ${ }^{\circ+}$ solution was then diluted with deionized water to yield an absorbance of 0.70 at $734 \mathrm{~nm}$. The ABTS ${ }^{\circ+}$ answer $(4 \mathrm{~mL})$ was added to $2 \mathrm{~mL}$ BSP solution at varying concentrations $(0.2,0.4$, $0.6,0.8,1.0,2.0,3.0,4.0$ and $5.0 \mathrm{mg} / \mathrm{mL}$ ). After $15 \mathrm{~min}$ of reaction, the absorbance was measured at $734 \mathrm{~nm}$. The ABTS ${ }^{\circ+}$ scavenging activity was concluded by the following equation:

Scavenging rate $(\%)=\left[1-\left(A_{1}-A_{2}\right) / A_{0}\right] \times 100 \%$

where $A_{0}$ is the absorbance of the control, $A_{1}$ is the absorbance of the BSP sample, and $A_{2}$ is the absorbance of all the reaction reagents (except BSP solution).

\section{Statistical analysis}

All experiments were performed three times, and SPSS 19.0 was used to analyze data. One-way analysis of variance (ANOVA) and Duncan's multiple range tests were used to determine the differences among experimental groups. $P<0.05$ was considered to be statistically significant, and $P<0.01$ was regarded as highly statistically significant.

\section{Results and Discussion}

\section{Single-factor experimental analysis}

In this study, the optimization of six key parameters, including liquid-solid ratio, extraction times, ethanol concentration, sample immersion time, extraction time and extraction temperature for improving the yield of BSP was investigated. The effect of extraction method on the yield was showed in Figure 1. The BSP yields of heating reflux extraction were higher than those of ultrasonic extraction at different extraction temperatures. Thus, the heating reflux extraction was selected for extraction of BSP.

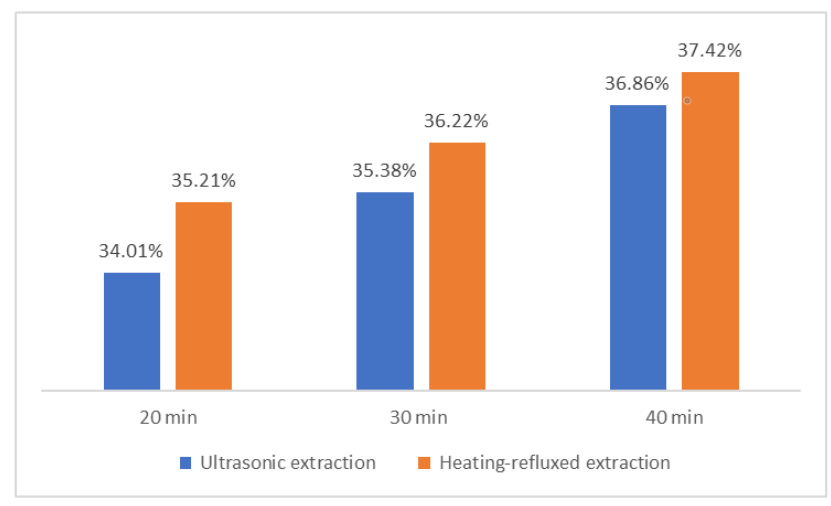

Figure 1 The effects of extraction methods on the yield.

The effect of liquid-solid ratio on the yield was showed in Figure 2A. The extraction yield of BSP gradually and slightly increased with the liquid-solid ratio increased from 15 to 25 $\mathrm{mL} / \mathrm{g}$, and then it tended to be constant from 25 to $30 \mathrm{~mL} / \mathrm{g}$. Large liquid-solid ratio can lead to a slightly increase of the 
Table 1 Box-Behnken experiment design and the BSP yield

\begin{tabular}{ccccc}
\hline Run & $A /{ }^{\circ} \mathrm{C}$ & $B / \min$ & $\mathrm{C} / \mathrm{min}$ & Yield of BSP/\% \\
\hline 1 & 90 & 120 & 60 & 46.05 \\
2 & 95 & 90 & 60 & 41.20 \\
3 & 85 & 60 & 90 & 42.74 \\
4 & 90 & 60 & 60 & 44.87 \\
5 & 95 & 90 & 43.47 \\
6 & 85 & 90 & 45.73 \\
7 & 90 & 120 & 90.11 \\
8 & 95 & 90 & 120 & 42.89 \\
9 & 90 & 90 & 90 & 48.38 \\
10 & 85 & 90 & 120 & 44.51 \\
11 & 90 & 90 & 120 & 46.77 \\
12 & 90 & 90 & 48.83 \\
13 & 90 & 90 & 47.98 \\
14 & 90 & 90 & 90 & 48.05 \\
15 & 95 & 90 & 90 & 43.82 \\
16 & 90 & 90 & 120 & 40.12
\end{tabular}
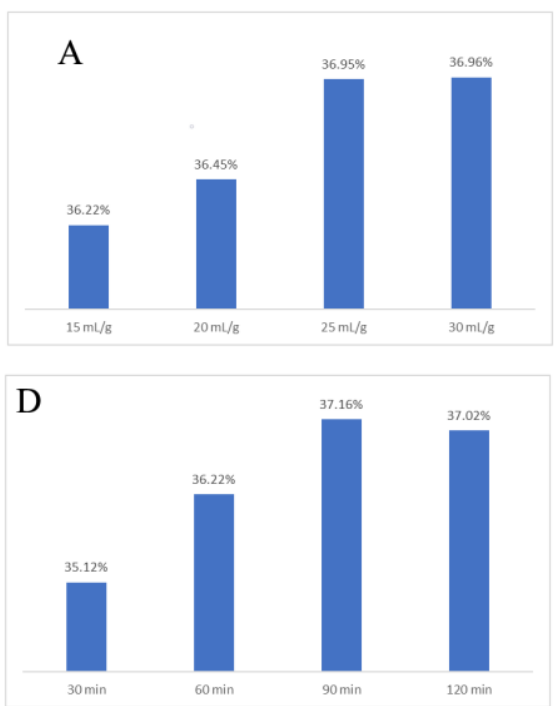

\section{B}
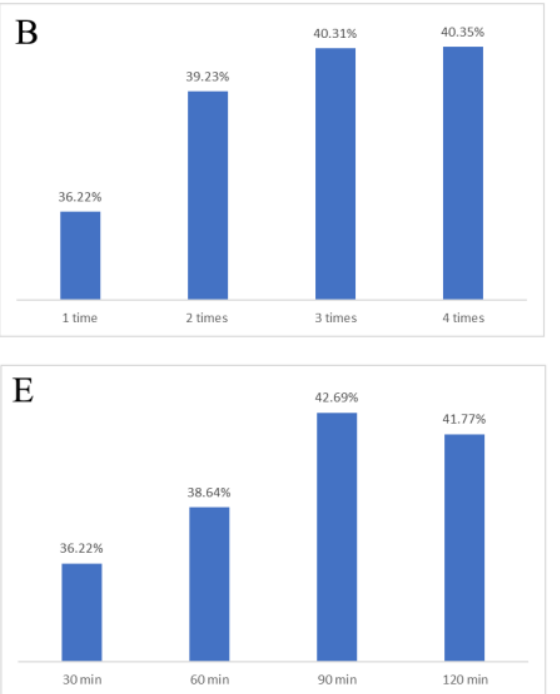

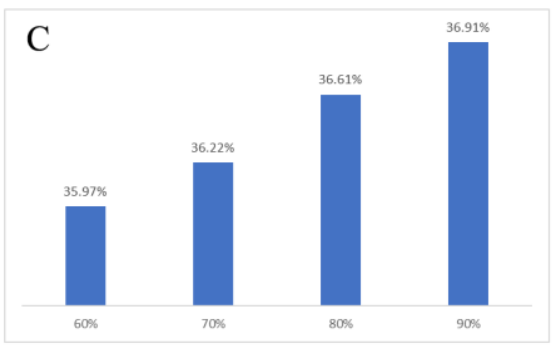

F

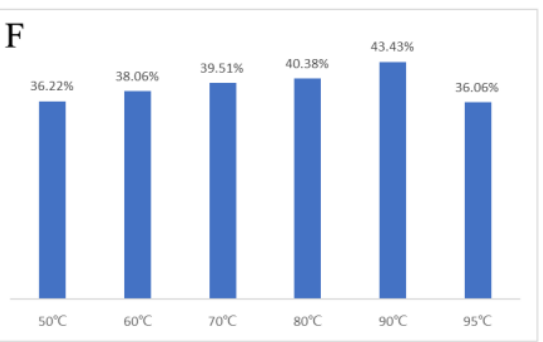

Figure 2 Effects of different experimental variables on the extraction yield of polysaccharides. (A) Liquid-solid ratio, (B) extraction times, (C) ethanol concentration, (D) sample immersion time, (E) extraction time, and (F) extraction and temperature.

extraction yield, and a large amount of extraction solvent can cause waste of energy and increase of workload. Therefore, the liquid-solid ratio of $15 \mathrm{~mL} / \mathrm{g}$ was selected as the fix parameter for further experiments.

The effect of extraction times on the yield was showed in Figure 2B. The extraction yield of BSP gradually increased with the extraction times from 1 to 3 . There was no significant increase when the extraction time was above 2. Therefore, the extraction time of 2 was selected as the fix parameter for further experiments.
The effect of ethanol concentration on the yield was showed in Figure $2 \mathrm{C}$. The extraction yield of BSP gradually and slightly increased with increase of ethanol concentration from $60 \%$ to $90 \%$, but it is indistinctive. A large amount of ethanol can cause the waste. It is not important, and the ethanol concentration of $70 \%$ was selected as the fix parameter for further experiments.

The effect of sample immersion time on the yield was showed in Figure 2D. The extraction yield of BSP gradually increased with increase of the sample immersion time from 30 
Table 2 Analysis of variance (ANOVA) for the quadratic model of BSP extraction

\begin{tabular}{|c|c|c|c|c|c|}
\hline Source & Sum of squares & df & Mean square & $F$-value & $P$-value \\
\hline Model & 107.75 & 9 & 11.97 & 20.68 & $0.0003^{* *}$ \\
\hline$A-X 1$ & 10.40 & 1 & 10.40 & 17.96 & $0.0039^{* *}$ \\
\hline$B-X 2$ & 6.90 & 1 & 6.90 & 11.92 & $0.0107^{\#}$ \\
\hline$C-x 3$ & 3.24 & 1 & 3.24 & 5.59 & $0.0500^{\#}$ \\
\hline$A B$ & 0.032 & 1 & 0.032 & 0.056 & $0.8198^{\#}$ \\
\hline$A C$ & 0.25 & 1 & 0.25 & 0.43 & $0.5321^{\#}$ \\
\hline $\mathrm{BC}$ & 0.40 & 1 & 0.40 & 0.70 & $0.4315^{\#}$ \\
\hline$A^{2}$ & 71.98 & 1 & 71.98 & 124.34 & $<0.0001^{* *}$ \\
\hline $\mathrm{B}^{2}$ & 5.12 & 1 & 5.12 & 8.84 & $0.0207^{*}$ \\
\hline$c^{2}$ & 4.31 & 1 & 4.31 & 7.45 & $0.0293^{*}$ \\
\hline Residual & 4.05 & 7 & 0.58 & & \\
\hline Lack of fit & 3.51 & 3 & 1.17 & 8.63 & $0.0321^{*}$ \\
\hline Pure error & 0.54 & 4 & 0.14 & & \\
\hline Cor Total & 111.81 & 16 & & & \\
\hline$R^{2}$ & 0.9638 & & & & \\
\hline Adjusted $R^{2}$ & 0.9172 & & & & \\
\hline Predicted $R^{2}$ & 0.4901 & & & & \\
\hline Adeq precsion & 12.673 & & & & \\
\hline C.V.\% & 1.68 & & & & \\
\hline
\end{tabular}

* Significant co-effect $(P<0.05) .{ }^{* *}$ High significant co-effect $(P<0.01) .{ }^{\#}$ Not significant $(P>0.05)$.

to $90 \mathrm{~min}$, while it decreased to $120 \mathrm{~min}$. Long large sample immersion time can lead to a slightly increase of extraction yield. Thus, the sample immersion time was selected for further research.

The effect of extraction time on the yield was showed in Figure 2E. The extraction yield of BSP gradually increased with the extraction time increased from 30 to $90 \mathrm{~min}$, while it decreased to $120 \mathrm{~min}$. Long large extraction time can lead to a slightly increase of the extraction yield and it is waste of time. Therefore, the extraction time was selected for further study.

The effect of extraction temperature on the yield was showed in Figure 2F. It is one of the important factors that influence the extraction yield of BSP. The extraction yield of BSP gradually increased with the extraction temperature increased from 50 to $90{ }^{\circ} \mathrm{C}$, while it decreased to $95{ }^{\circ} \mathrm{C}$. Too high extraction temperature is not conducive to the extraction of BSP. Therefore, the extraction temperature was selected for further study.

\section{Fitting the model}

Based on the results of single factor experiments, extraction temperature, extraction time and sample immersion time were further investigated using BBD to obtain the optimal extraction conditions. The design matrix and results were shown in Table 1. The response variable $Y$ for extraction yield of BSP can be described by the following equation:

$$
\begin{aligned}
Y= & 48.25-1.14 X_{1}+0.93 X_{2}+0.64 X_{3}+0.090 X_{1} X_{2}+0.25 X_{1} X_{3} \\
& -0.32 X_{2} X_{3}-4.13 X_{1}^{2}-1.10 X_{2}^{2}-1.01 X_{3}^{2}
\end{aligned}
$$

where $Y$ is the BSP yield; $X_{1}, X_{2}$ and $X_{3}$ are the factors of extraction temperature, extraction time and sample immersion time, respectively.

The analysis of variance (ANOVA) and adequacy of quadratic model were shown in Table 2. The importance of coefficients can be verified by their respective $P$-values, and smaller $P$-values signifies a more significant comparing coefficient. In Table 2, the model $F$-value of 20.68 implies the model in significance. The model $P$-value was only $0.03 \%$ that demonstrates the relapse display for BSP yield was highly significant. Furthermore, the values of determination coefficient $\left(R^{2}=0.9638\right)$, adjusted determination coefficient (adjusted $R^{2}=$ 0.9172 ) and low coefficient of variance (C.V. $=1.68)$ verified the significance of regression models and the accuracy of experimental data. The linear co-efficient $(A)$, two quadratic coefficients $\left(B^{2}\right.$ and $\left.C^{2}\right)$ had a significant effect on the yield $(P<$ $0.05)$, while the other interaction coefficients $(A B, A C, B C$ and $A^{2}$ ) were not significant.

\section{Analysis of response surface}

Figure 3 showed 3D response surface plots and their corresponding contour plots about the reciprocal interactions between two independent extraction parameters. As shown in Figures $3 \mathrm{~A}-3 \mathrm{D}$, when extraction time and sample immersion time were designated at level 0 , the BSP yield increased firstly with increase of extraction temperature, and then it decreased. When extraction temperature was fixed at level 0, the BSP yield increased with increase of extraction time and sample immersion time. The reason might be that excessive extraction temperature could also cause the degradation of polysaccharides. 
A

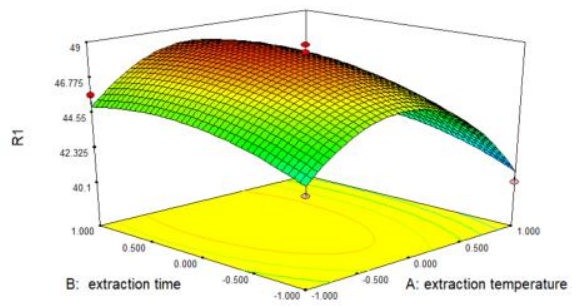

D

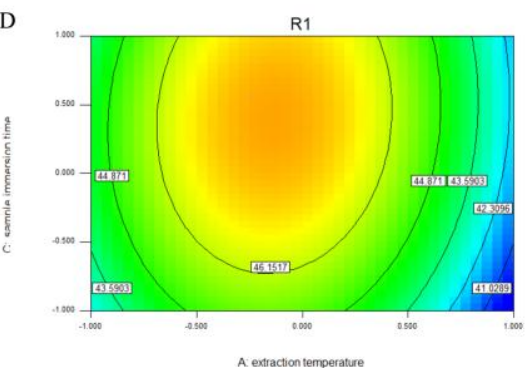

B
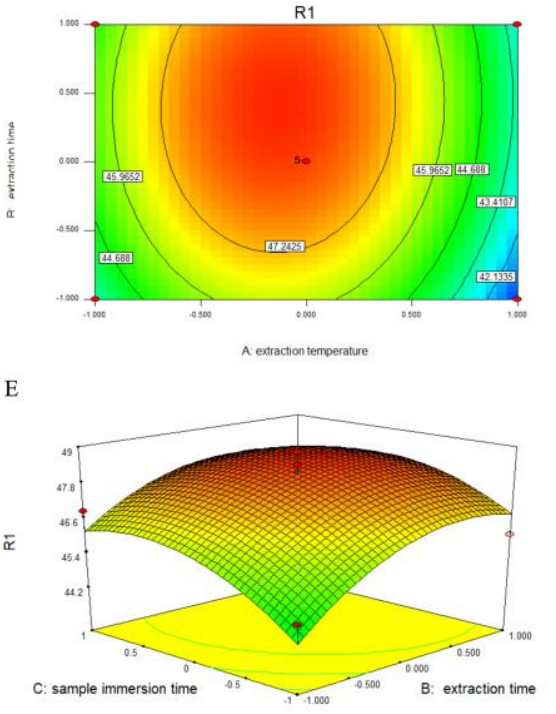
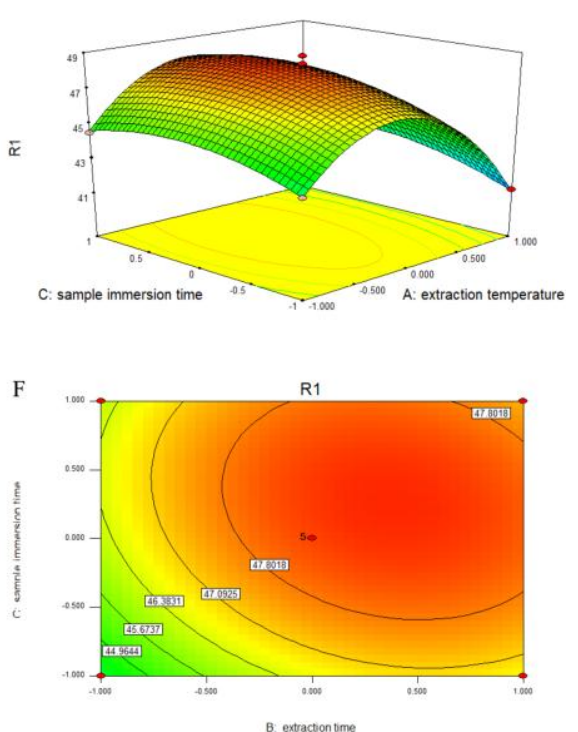

Figure 3 Response surface and contour plots for \% yield of BSP. (A, B) extraction temperature and extraction time, (C, D) extraction temperature and sample immersion time, (E, F) extraction time and sample immersion time.

A

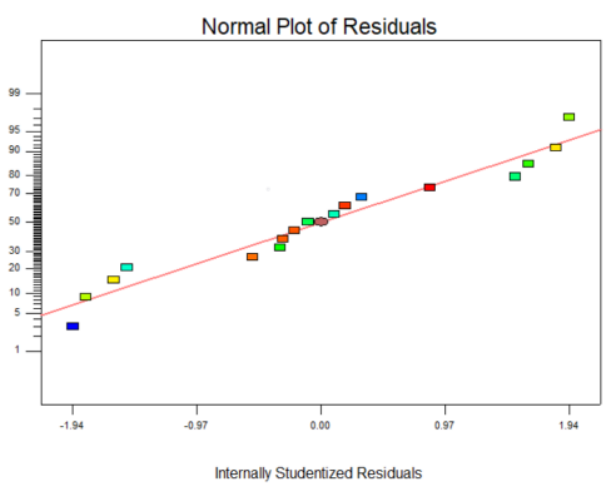

$\mathrm{C}$

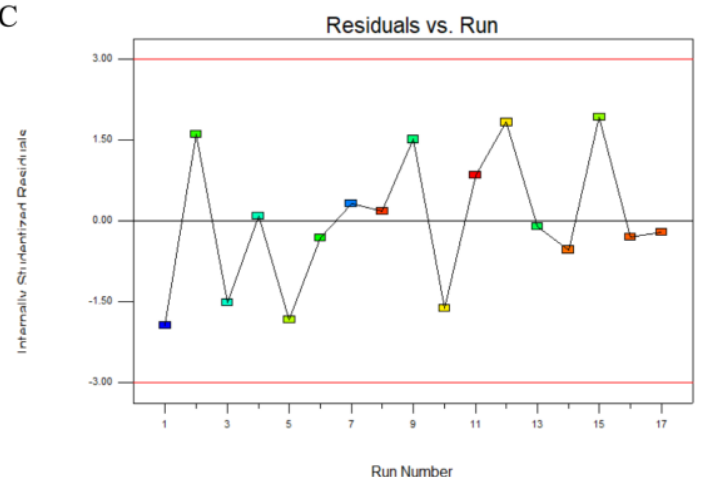

B

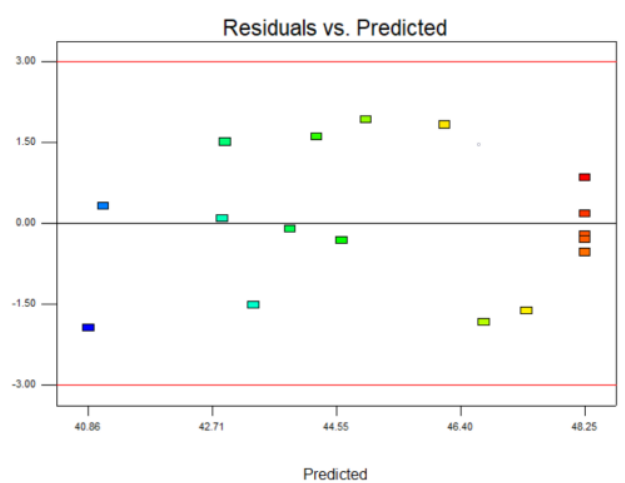

D

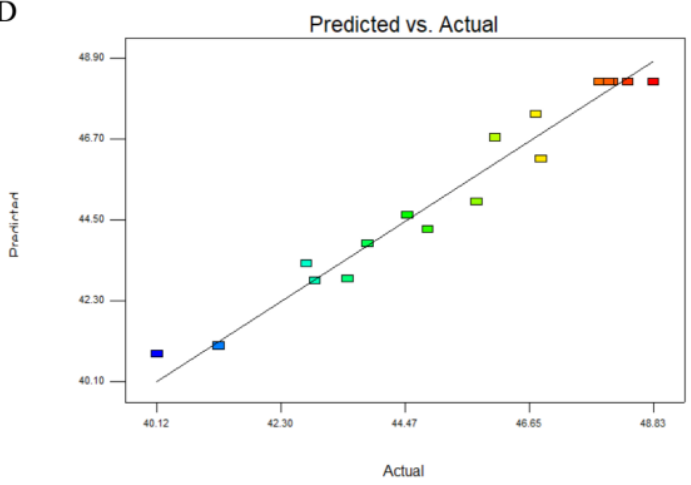

Figure 4 Diagnostic plots for the Box-Behnken model adequacy. (A) Normal plot of residuals, (B) plot of internally studentized residuals vs. predicted response, (C) residual vs. run, and (D) predicted $v s$. actual.

From Figures $3 E$ and $3 F$, a significant increase of the polysaccharides yield was observed with increase of sample immersion time and extraction time. These two variables had positive reciprocal action on response of extraction yield, however, long extraction time and sample immersion time could not increase the extraction ratio of polysaccharides. The optimum conditions for the BSP yield were obtained as follows: the extraction time of $90 \mathrm{~min}$, sample immersion time of $90 \mathrm{~min}$, and the extraction temperature of $90^{\circ} \mathrm{C}$. Under these optimal conditions, the maximum extraction yield of BSP was close to the predicted value. These findings indicated that the adequacy of model equation was confirmed, and the optimizing extraction process was suitable for BSP (Figure 4). 


\section{Antioxidant activity in vitro}

DPPH radical is a stable free-radical, which can accept an electron or a hydrogen atom from the antioxidant scavenger molecule to be converted to a more stable DPPH molecule. It is widely used to evaluate the free radical scavenging ability of antioxidants. Studies have shown that polysaccharides exhibited potent antioxidant capacity. Hence, the antioxidant activity of BSP was investigated shown in Figure 5. BSP showed a highest scavenging effect on $\mathrm{ABTS}^{\circ+}$ with scavenging rate of $65.34 \%$ at the concentration of $5 \mathrm{mg} / \mathrm{mL}$. Meanwhile, it lowered the scavenging effect on DPPH radical relatively $(48.15 \%)$, which was correlated positively with increasing concentrations. At the low concentration ranges, the scavenging rate and the concentration of BSP are near linear. However, Vc showed scavenging rates on $\mathrm{ABTS}^{*+}$ and DPPH radical of $100 \%$. The results implied that BSP has a slight free radical scavenging ability of antioxidants.

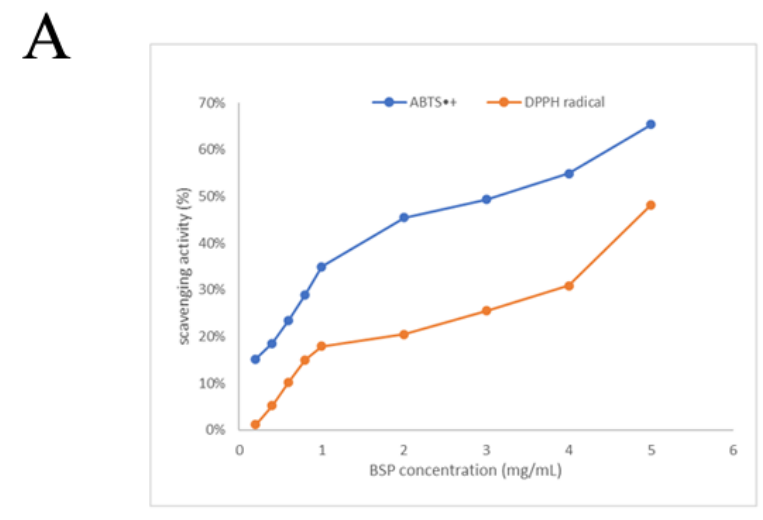

\section{B}

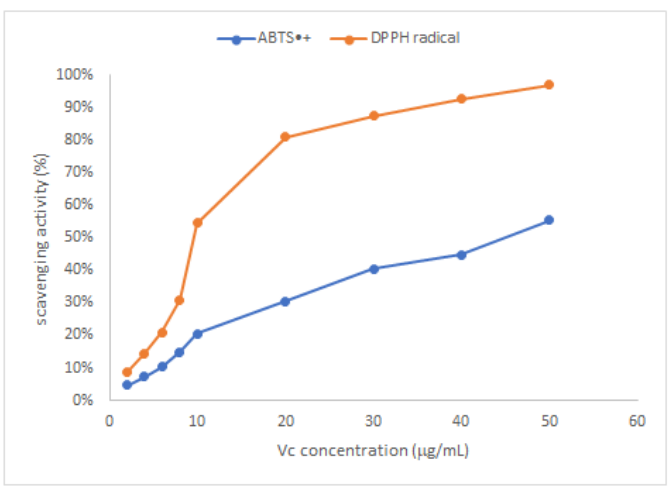

Figure 5 Antioxidant activity in vitro. (A) Scavenging activity of $V_{c}$ to DPPH and $A_{B T S}{ }^{\circ+}$ from 0 to $50 \mathrm{ug} / \mathrm{mL}$, and (B) scavenging activity of $V_{c} B S P$ to DPPH and ABTS from 0 to $5 \mathrm{mg} / \mathrm{mL}$.

\section{Conclusion}

In this paper, BSP was obtained using conventional hot water extraction and ethanol precipitation from Bletilla striata. The maximum yield of polysaccharides was $48.38 \%$ under the optimum conditions of liquid-solid ratio of $15 \mathrm{mg} / \mathrm{mL}$, extraction temperature $90^{\circ} \mathrm{C}$, extraction time of $90 \mathrm{~h}$, sample immersion time $90 \mathrm{~min}$, and extraction two times. In addition, the antioxidant assays in vitro revealed that BSP has a slight free radical scavenging ability of antioxidants and a scavenging effect of BSP on ABTS ${ }^{++}$with scavenging rate of $65.34 \%$ at the concentration of $5 \mathrm{mg} / \mathrm{mL}$. However, it lowers the scavenging effect on DPPH radical (48.15\%) relatively. The scavenging effect of BSP on $\mathrm{ABTS}^{*+}$ was higher than $\mathrm{DPPH}$ radical.

\section{Acknowledgements}

This work was financially supported by the Provincial Project "Construction of Quality Control System for Genuine Chinese Medicinal Materials in Yunnan (No. 2017AB005)" from Yunnan Major Scientific and Technological Projects.

\section{Author Contributions}

X. S. performed the experiments and wrote a draft of the manuscript. W. J. checked the draft, supervised the work and edited the final version of the manuscript.

\section{Conflict of Interest}

The authors declare no conflict of interest.

Copyright (c) 2020 Xiaojing Shen and Weiwei Jiang. This article is an open access article distributed under the terms and conditions of the Creative Commons Attribution (CC BY) license (http://creativecommons. org/licenses/by/4.0/). The use, distribution or reproduction in other forums is permitted, provided the original author(s) or licensor are credited and that the original publication in this journal is cited, in accordance with accepted academic practice. No use, distribution or reproduction is permitted which does not comply with these terms.

\section{References}

[1] Bulpitt, C. J.; Li, Y.; Bulpitt, P. F.; Wang, J. The use of orchids in Chinese medicine. J. Royal Soc. Med. 2007, 100, 558-563.

[2] Jiang, F.; Li, M.; Wang, H. Coelonin, an anti-inflammation active component of Bletilla striata and Its Potential Mechanism. Int. J. Mol. Sci. 2019, 20, 4422

[3] Jiang, S.; Chen, C. F.; Ma, X. P. Antibacterial stilbenes from the tubers of Bletilla striata. Fitoterapia 2019, 138, 104350

[4] Liu, B.; Zhan, Q.; Wu, X. Effect of Bletilla striata on the prevention of postoperative peritoneal adhesions in abrasion-induced rat model. Evid. Based. Complement. Alternat. Med. 2019, 9148754.

[5] Zhou, H.; Jin, Y.; Gu, C. Bletilla striata promotes the healing of enterocutaneous fistula: A case report. Medicine (Baltimore) 2019, 98, e16288.

[6] Jiang, F.; Li, W.; Huang, Y. Antioxidant, antityrosinase and antitumor activity comparison: the potential utilization of fibrous root part of Bletilla striata (Thunb.) Reichb.f. PLoS One 2013, 8, e58004.

[7] Qian, C. D.; Jiang, F. S.; Yu, H. S.; Ding, Z. S. Antibacterial Biphenanthrenes from the fibrous roots of Bletilla striata. J. Nat. Prod. 2015, 78, 939-943.

[8] Shi, Y.; Zhang, B.; Lu, Y. Antiviral activity of phenanthrenes from the medicinal plant Bletilla striata against influenza a virus. BMC Complement. Altern. Med. 2017, 17, 273.

[9] He, X.; Wang, X.; Fang, J. Bletilla striata: Medicinal uses, phytochemistry and pharmacological activities. J. Ethnopharmacol. 2017, 195, 20-38.

[10] Chen, Z.; Zhao, Y.; Zhang, M. Structural characterization and antioxidant activity of a new polysaccharide from Bletilla striata fibrous roots. Carbohydr. Polym. 2020, 227, 115362.

[11] Luo, L.; Liu, Y.; Cai, X. Bletilla striata polysaccharides ameliorates lipopolysaccharide-induced injury in intestinal epithelial cells. Saudi. J. Gastroenterol. 2019, 25, 302-308.

[12] Wang, Y.; Han, S.; Li, R. Structural characterization and immunological activity of polysaccharides from the tuber of Bletilla striata. Int. J. Biol. Macromol. 2019, 122, 628-635.

[13] Yue, L.; Wang, W.; Wang, Y. Bletilla striata polysaccharide inhibits angiotensin II-induced ROS and inflammation via NOX4 and TLR2 pathways. Int. J. Biol. Macromol. 2016, 89, 376-388.

[14] Yu, J.; Hu, M.; Wang. Y. Extraction, partial characterization and 
bioactivity of poly saccharides from Senecio scandens Buch. Ham. Int. J. Biol. Macromol. 2018, 109, 535-543.

[15] Gong, G.; Dang, T.; Deng, Y. Physicochemical properties and biologic activities of polysaccharides from Lycium barbarum prepared by fractional precipitation. Int. J. Biol. Macromol. 2018, 109, 611-618.

[16] Wang, Y.; Jia, J.; Ren, X. Extraction, preliminary characterization and in vitro antioxidant activity of polysaccharidies from Oudemansiella dadicata mushroom. Int. J. Biol. Macromol. 2018, 120, 1760-1769.

Received May 25, 2020

Accepted June 16, 2020 